Ikonion Journal of Mathematics
https://dergipark.org.tr/tr/pub/ikjm
Research Article
Open Access

\title{
Embedding Orthomorphisms d-Algebra in Biorthomorphisms as Ordered Ideal
}

\author{
İbrahim Gökcan1 ${ }^{1}$ (1)
}

\author{
Keywords: \\ d-Algebra, \\ Biorthomophism, \\ Ordered ideal, \\ Orthomorphism.
}

\begin{abstract}
In the historical development of Riesz spaces, we can trace the history of ordered vector spaces to the International Mathematical Congress in Bologna in 1928. Studies related with $f$-Algebras for the Dedekind complete ordered vector space defined in Riesz spaces were initiated by Nakano and their current definition was made by Amemiya, Birkhoff and Pierce.The revival of $f$-algebras, which had a tendency to slow down for a period of time, emerged as a result of Pagter's doctoral thesis [9] and the examination of Alkansas lecture notes by Luxemburg. The concepts of homomorphism, isomorphism, orthomorphism and biorthomorphism in Riesz spaces are defined by Zaanen, Huijsmans, Boulabiar, Buskes and Triki. Algebraic structure of biorthomorphisms defined on Riesz space examined by [8]. $f$-Algebra on $\operatorname{Orth}(X, X)$ were studied by [8] and [6]. [6] demonstrated that biorthomorphisms space have an $f$-algebraic structure with the help of the product defined as $\left(T_{1} *_{\mathrm{e}} T_{2}\right)(x, y)=T_{1}\left(x, T_{2}(e, y)\right)$ for $e \in X^{+}, \forall x, y \in X$ and $T_{1}, T_{2} \in \operatorname{Orth}(X, X)$. [8] showed that if $X$ are semiprime Dedekind complete $f$-algebras, $\operatorname{Orth}(X)$ is an ordered ideal in biorthomorphisms. [6] developed an alternative proof for this situation. If $X$ Archimedean Riesz space, $\operatorname{Orth}(X)$ is an $f$-algebra according to compound operation with unit element. [11] showed that if $X$ is a semiprime $f$-algebra, it is a $d$-algebra. In this study, we investigated embedding orthomorphism in biorthomorphisms when $X$ is uniformly complete $d$-algebra.
\end{abstract}

Subject Classification (2020): 06B05, 06B10.

\section{Introduction}

In this section, we gave some definitions about Riesz space and Riesz algebra.

Definition 1. Let $X$ is a set different from empty. If relation $\leq$ defined on $X$ satisfy the following properties, relation $\leq$ is called the (partial) order relation, the pair $(X, \leq)$ is called the (partial) ordered set. For $\forall x, y, z \in X$

i. $x \leq x$ for $\forall x \in X$.

ii. If $x \leq y$ and $y \leq x, x=y$.

iii. If $x \leq y$ and $y \leq z, x \leq z[1]$.

1 gokcan@artvin.edu.tr (Corresponding Author)

1Faculty of Science and Arts, Artvin Çoruh University, Artvin, Turkey

Article History: Received: 18.11.2021 — Accepted: 21.12.2021 — Published: 26.12.2021 
Definition 2. Let $(X, \leq)$ is an ordered vector space. If every finite subset different from empty has a supremum, $X$ is called a Riesz space (or a vector lattice). Supremum of $\{x, y\}$ is demonstrated with $x \vee y$ in classic Riesz space as notation [1].

Definition 3. Let $X$ is an Riesz space. If $n x \leq y \Rightarrow x=0$ is satisfy for $\forall n \in \mathbb{N}$ where $x, y \in X^{+}, X$ is called Archimedean Riesz space [1].

Definition 4. A Riesz space is called Dedekind complete if every non-empty upper bound (bottom bounded) subset has a supremum (infimum) [1].

Definition 5. Let $X$ is an Riesz space. If $X$ is an associative algebra and $x y \in X^{+}$for $\forall x, y \in X^{+}, X$ is called Riesz algebra (or ordered lattice algebra) [1].

Definition 6. Let $X$ is an Riesz algebra. If $x \cdot x=x^{2} \in X^{+}$for $\forall x \in X, X$ is called positive square or $X$ have the positive square property.

Definition 7. Let $X$ is an Riesz algebra. If $c x \wedge y=x c \wedge y=0$ is satisfy for $\forall x, y \in X, x \wedge y=0$ and $\forall c \in$ $X^{+}, X$ is called $f$-algebra [4].

Definition 8. Let $X$ is an Riesz algebra. If $x y=0$ is satisfy for $\forall x, y \in X, x \wedge y=0, X$ is called almost $f$-algebra [5].

Definition 9. Let $X$ is an Riesz algebra. If $c x \wedge c y=x c \wedge y c=0$ is satisfy for $\forall x, y \in X, x \wedge y=0$ and $\forall \mathrm{c} \in X^{+}, X$ is called $d$-algebra [12].

For more information about $d$-algebra, [3] can be given as reference.

Definition 10. Let $X$ is an Riesz algebra. If $x=0$ when $x^{k}=0$ for $x \in X$ and $\exists k \in \mathbb{N}, X$ is called semi prime [1].

Theorem 1. If $X$ is a semiprime $f$-algebra, the following statements are are equivalent to each other.

i. $X$ is a $f$-algebra.

ii. $X$ is a $d$-algebra.

iii. $X$ is an almost $f$-algebra [11].

Definition 11. If the operator $T$ transforms every ordered bounded subset of $X$ to an ordered bounded subset of $Y$, where $X$ and $Y$ are ordered bounded vector spaces, transformation $T$ is called an ordered bounded operator. The set of all ordered bounded operators is denoted by $L_{b}(X, Y)$ [1].

[13] can be examined about biliner operators.

Definition 12. Let $X$ is an Riesz space. If $g \in S$ for $|g| \leq|f|$ where $S \subseteq X, g \in X$ and $f \in S, S$ is called solid [1]. 
Definition 13. Let $X$ is an Riesz space and $Y \subseteq X$. If $Y$ is a solid linear subspace, $Y$ is called ideal in $X$.

If $A$ and $B$ are ideals in $Y, A \cap B$ and $A+B$ are ideals in $Y$. $A \cap B$ is an ideal in $Y$ because of $A \cap B \subseteq Y$ for $A \subseteq Y$ and $B \subseteq Y . A+B=\{A \cup B: A \cap B=\emptyset, A, B \in Y\}$ is an ideal in $Y$ because of $A+B \subseteq A \cup B \subseteq$ $Y[1]$.

Definition 14. Let $X$ is an Riesz space and $Y$ is an ideal of $X$.If any subset of $Y$ has a supremum in $X$ and this supremum is an element of $Y$, in other words, if $f \in Y$ is satisfied when $Z \subseteq Y$ and $f=\sup Z$, then the ideal $Y$ is called band in $X$ [14].

Definition 15. Let $X$ is an Riesz space and $T: X \rightarrow X$ is an linear operator. If $T(B) \subseteq B$ for $\forall B \subseteq X$, in other words if operator T leaves all bands of $X$ unchanged, $T$ is called band preserving operator [1].

Definition 16. Let $X$ an $Y$ are two Riesz spaces and $T: X \rightarrow Y$ is an linear operator. For $\forall x, y \in X$, if operator $T$ is satisfy $T(x \vee y)=T x \vee T y$, operator $T$ is called Riesz homomorphism [1].

Definition 17. A band preserving ordered bounded operator is called an orthomorphism. So, let $X$ is a Riesz space and $T: X \rightarrow X$ is an bounded operator. In $X, T x \perp y$ is provided when $x \perp y$. Also, if the orthomorphism $T$ is positive at the same time, $T$ is called a positive orthomorphism. In other words, $T$ is a positive orthomorphism if and only if $x \wedge y=0$ then $T x \wedge y=0$ on $X$. The set of all orthomorphisms on $X$ is denoted by $\operatorname{Orth}(X)[1]$.

Definition 18. Let $X$ is an Archimedean Riesz space. If bilinear transformation $T: X \times X \rightarrow X$ is an orthomorphism in each component of $X, T$ is called biorthomorphism. In other words, if $T(x,),. T(., x) \in$ $\operatorname{Orth}(X)$ for $\forall x \in X$, bilinear transformation $T: X \times X \rightarrow X$ is called biorthomorphism on $X$. The set of all biorthomorphism on $X$ is denoted by $\operatorname{Orth}(X, X)$.

Note. $\rho: \operatorname{Orth}(X) \rightarrow \operatorname{Orth}(X, X)$ defined with $\rho(T)(x, y)=T(x y)=T(x) y$ is one-to-one Riesz homomorphism for $\forall T \in \operatorname{Orth}(X)$ and $(x, y) \in X \times X[8]$.

$$
\begin{gathered}
\rho:(\operatorname{Orth}(X), X \times X) \rightarrow \operatorname{Orth}(X, X) \\
(T,(x, y)) \rightarrow \rho(T)(x, y)=T(x y)=T(x) y .
\end{gathered}
$$

Notation. $K(T)=\{x \in X: T(x, x)=0\}$ for $\forall T \in \operatorname{Orth}(X, X)[6]$.

Lemma 1. Let $X$ is an Archimedean Riesz space and $T \in \operatorname{Orth}(X, X)$.

$$
K(T)=\{x \in X: T(x, y)=0, \forall y \in X\} .
$$

Specially, $K(T)$ is an ordered ideal in $X[6]$.

Lemma 2. Let $X$ is an Archimedean Riesz space and $T \in \operatorname{Orth}(X, X)$. If $x \in X, T(x, x)=0 \Leftrightarrow T(x, x) \in$ $K(T)[6]$.

\section{Proposition 1.}

i. If $X$ is a semiprime $f$-algebra, $\operatorname{Orth}(X)$ is Riesz space in $\operatorname{Orth}(X, X)$.

ii. If $X$ is a semiprime Dedekind complete $f$-algebra, $\operatorname{Orth}(X)$ is an ordered ideal in $\operatorname{Orth}(X, X)[8]$. 
Theorem 2. Let $X$ is a semiprime Dedekind complete $f$-algebra. $\rho(\operatorname{Orth}(X))$ is an ordered ideal in $\operatorname{Orth}(X, X)[2]$.

\section{Embedding Orthomorphisms in Biorthomorphisms}

Let $X$ is a semiprime $f$-algebra. Specially, $x T(y)=T(x y)=y T(x)$ is provided for $\forall x, y \in X$ and $T \in$ $\operatorname{Orth}(X)$ from $\rho: \operatorname{Orth}(X) \rightarrow \operatorname{Orth}(X), \rho(\pi)(x, y)=\pi(x y)=\pi(x) y$ for $\forall \pi \in \operatorname{Orth}(X)$ and $(x, y) \in$ $X \times X$. If transformation $\widehat{T}: X \times X \rightarrow X$ is satisfy $\widehat{T}(x, y)=T(x y)=T(x) y, \widehat{T}$ is called biorthomorphism. Therefore, if $\rho(T)(x, y)=\widehat{T}(x, y), \rho(T)=\widehat{T}$. Transformation $\rho: \operatorname{Orth}(X) \rightarrow \operatorname{Orth}(X, X)$ is an one-to-one Riesz homomorphism [8]. So, $\operatorname{Orth}(X)$ as Riesz subspace is embedded in $\operatorname{Orth}(X, X)$ under transformation $\rho$. Then, $T \in \operatorname{Orth}(X)$ determined with $\widehat{T} \in \operatorname{Orth}(X, X)$.

Theorem 3. If $X$ Riesz space is uniformly complete, $\operatorname{Orth}(X, X)$ is uniformly complete [6].

\subsection{Embedding Orthomorphisms in Biorthomorphisms when $X$ is Uniformly Complete Semiprime $f$-Algebra}

Let $X$ is an uniformly complete semiprime $f$-algebra and $X^{\odot}=\{x y: x, y \in X\}$. Then the set $X^{\odot}$ is a Riesz subspace of $X$ with positive cone $\left\{x x=x^{2}: x \in X\right\}$ [7].

Theorem 4. Let $X$ is an uniformly complete semiprime $f$-algebra and transformation $T: X \times X \rightarrow X . T$ is a (positive) biorthomorphism on $X \Leftrightarrow$ There is only one positive biorthomorphism $T^{\odot}: X^{\odot} \rightarrow X$ satisfying property $T(x, y)=T^{\odot}(x y)$ for $\forall x, y \in X[6]$.

Proof. $\Rightarrow$ Let $T$ is a biorthomorphism on $X$. Let positive biorthomorphism $T^{\odot}: X^{\odot} \rightarrow X$ is satisfy property $T(x, y)=T^{\odot}(x y)$ for $\forall x, y \in X$. Let us now show that there is only one biorthomorphism. Let $T_{1}{ }^{\odot}$ ve $T_{2}{ }^{\odot}$ are two biorthomorphisms satisfying $T(x, y)=T^{\odot}(x y)$ on $X$ for $\forall x, y \in X . T_{1}{ }^{\odot}(x y)=$ $T_{1}(x, y)=T_{1}(x y)$ is provided for transformation $T_{1}{ }^{\odot}: X^{\odot} \rightarrow X$. Similarly, $T_{2}{ }^{\odot}(x y)=T_{2}(x, y)=T_{2}(x y)$ is provided for $T_{2}{ }^{\circ}: X^{\odot} \rightarrow X$. If $T_{1}(x y)=T_{2}(x y), T_{1}=T_{2}$. Then $T_{1}{ }^{\odot}=T_{2} \odot$.

$\Leftarrow$ If $T$ is a positive biorthomorphism on $X, T$ is called orthosymmetric Riesz bimorphism. Transformation $\odot: X \times X \rightarrow X$ defined with $\odot(x, y)=x y$ is orthosymmetric Riesz bimorphism, $\left(X^{\odot}, \odot\right.$ ) is a Riesz space and square of $X$ [7]. Therefore, there is an only Riesz homomorphism $T^{\odot}: X^{\odot} \rightarrow X$ defined with $T(x, y)=T^{\odot}(x y)$ for $\forall x, y \in X$. Moreover $T^{\odot}$ is an orthomorphism. Indeed, let $|x| \wedge|v|=$ 0 for $x \in X$ and $v \in X^{\odot} . T(x, v)=0$ from $T$ orthosymmetric. On the other hand, there are $y, z \in X$ satisfying property $v=y z$ from definition $X^{\odot}$ for $v \in X^{\odot}$. From $\rho(\pi)(x, y)=\pi(x y)=\pi(x) y$ for $\forall \pi \in$ $\operatorname{Orth}(X)$ and $(x, y) \in X \times X$, then $x T(y, z)=T(x y, z)=y T(x, z)=T(x, y z)=T(x, v)=0$ for $T \in$ $\operatorname{Orth}(X, X)$. Since $X$ is semiprime,

$$
|x| \wedge\left|T^{\odot}(v)\right|=|x| \wedge\left|T^{\odot}(y z)\right|=|x| \wedge|T(y, z)|=0 .
$$

This demonstrated that operator $T^{\odot}$ is a positive orthomorphism.

Conclusion 1. Let $X$ is Dedekind complete semiprime $f$-algebra. $\operatorname{Orth}(X)$ is an ordered ideal of $\operatorname{Orth}(X, X)[6]$. 
Proof. Since $\operatorname{Orth}(X)$ is a Riesz subspace of $\operatorname{Orth}(X, X)$, it is sufficient to prove the theorem to show that $\operatorname{Orth}(X)$ is a solid in $\operatorname{Orth}(X, X)$. For this let $T \in \operatorname{Orth}(X, X)$. By definition of solid, let $0 \leq T \leq f$ for $f \in$ $\operatorname{Orth}(X)$. Here we have to show that $T \in \operatorname{Orth}(X)$. Since $X$ is uniformly complement, there is an only positive orthomorphism $T^{\odot}: X^{\odot} \rightarrow X$ satisfying $T(x, y)=T^{\odot}(x y)$ for $\forall x, y \in X$. From here,

$$
0 \leq T^{\odot}\left(x^{2}\right)=T^{\odot}(x x)=T(x, x) \leq f\left(x^{2}\right)
$$

is provided. In other words, $0 \leq T^{\odot}(v) \leq f(v)$ is provided for $\forall 0 \leq v=x^{2} \in X^{\odot}$. The operator $T^{\odot}$ has an extension to a positive operator that satisfies the property $0 \leq T^{\odot} \leq f$, which we can denote again with $T^{\odot}$. From here, $T^{\odot} \in \operatorname{Orth}(X)$ and $\widehat{T \odot}=T$ is obtained. Consequently, $T \in \operatorname{Orth}(X)$ is found. This proves that $\operatorname{Orth}(X)$ is a solid in $\operatorname{Orth}(X, X)$ and therefore an ordered ideal.

\subsection{Embedding Orthomorphism in Biorthomorphisms when $X$ is Uniformly Complete Semiprime $f$-Algebra with Weak Ordered Unit}

Theorem 5. If $X$ is uniformly complete semiprime $f$-algebra with weak ordered unit, $\operatorname{Orth}(X)$ is an ordered ideal in $\operatorname{Orth}(X, X)[6]$.

Proof. $\operatorname{Orth}(X, X)$ is an uniformly complement semiprime $f$-algebra. Let $e \in X$ is an positive weak ordered unit. $\left(\operatorname{Orth}(X, X), *_{e}\right)$ is an semiprime $f$-algebra [6]. Let us show that $\operatorname{Orth}(X)$ is a ring ideal in $\left(\operatorname{Orth}(X, X), *_{e}\right)$. Let $f \in \operatorname{Orth}(X)$ and $T \in \operatorname{Orth}(X, X)$. From here, $\left(\hat{f} *_{e} T\right)(x, y)=\hat{f}(x, T(e, y))=$ $f(x T(e, y))=x f(T(e, y))=x(f \mathrm{o} T(e,)).(y)=f \mathrm{o} \widehat{T(e, .})(x, y)$ is provided from $x f(y)=f(x y)=y f(x)$ for $f$ oT $(e,.) \in \operatorname{Orth}(X)$ and $\forall x, y \in X$. Then, $\left.\hat{f} *_{e} T=f o \widehat{(e,}.\right) \in \operatorname{Orth}(X)$. This shows that $\operatorname{Orth}(X)$ is a ring ideal in $\operatorname{Orth}(X, X)$. On the other hand, since $\operatorname{Orth}(X)$ is an uniformly complete $f$-algebra with unit element, $\operatorname{Orth}(X)$ is square root closed. At the same time, ring ideal $\operatorname{Orth}(X)$ is an ordered ideal. From this, it is concluded that $\operatorname{Orth}(X)$ is an ordered ideal in $\operatorname{Orth}(X, X)$.

\subsection{Embedding Orthomorphism in Biorthomorphisms when $X$ is Uniformly Complete $d$-Algebra}

In this section, we defined the subspace $X^{d}=\left\{c x \wedge c y: x \wedge y=0, c \in X^{+}, x, y \in X\right\}$ of $X$, which is an $d$-algebra. Following conclusion is obtained from Theorem 1 .

Conclusion 2. Let $X$ is an uniformly complete $d$-algebra. For $c \in X^{+}, x, y \in X$;

i. If $y=x, X^{d}=X^{\odot}$.

ii. If $y=x=c$ then set $X^{d}$ is a Riesz subspace of space $X$ with positive cone $\left\{c c=c^{2}: c \in X\right\}$.

\section{Proof.}

i. $X^{d}=\left\{c x \wedge c y: x \wedge y=0, c \in X^{+}, x, y \in X\right\}$

$$
\begin{aligned}
& =\left\{c x \wedge c x: x \wedge x=0, c \in X^{+}, x \in X\right\}(y=x) \\
& =\left\{c x: c \in X^{+}, x \in X\right\}
\end{aligned}
$$

From here, $X^{d}=X^{\odot}$ is provided.

ii. $X^{d}=\left\{c x \wedge c y: x \wedge y=0, c \in X^{+}, x, y \in X\right\}$

$$
=\left\{c c \wedge c c: c \wedge c=0, c \in X^{+}\right\}(y=x=c)
$$




$$
=\left\{c^{2}: c \in X^{+}\right\}
$$

From here, $X^{d}$ is a Riesz subspace of $X$ with positive cone $\left\{c c=c^{2}: c \in X\right\}$.

Conclusion 3. Let $X$ is uniformly complete $d$-algebra, $T: X \times X \rightarrow X$ is an transformation and $T$ is an biorthomorphism on $X$. For $\forall x, y \in X$ and $c \in X^{+}$;

i. If $y=x$, there is an only positive biorthomorphism $T^{d}: X^{d} \rightarrow X$ satisfying property $T(c, x)=T^{d}(c x \wedge c y)$. ii. If there is an only biorthomorphism $T^{d}: X^{d} \rightarrow X$ satisfying property $T(c, x)=T^{d}(c x \wedge c y)$ for $y=x, T$ is a positive biorthomorphism on $X$.

iii. If $y=x$ for $\forall x, y \in X$ and $c \in X^{+}, T^{\odot}=T^{d}$.

\section{Proof.}

i. Let $y=x, T_{1}^{d}$ and $T_{2}^{d}$ are two biorthomorphisms satisfying transformation $T^{d}: X^{d} \rightarrow X$.

$$
\begin{gathered}
T_{1}^{d}(c x \wedge c y)=T_{1}^{d}(c x \wedge c x)=T_{1}^{d}(c x)=T_{1}(c, x) \\
T_{2}^{d}(c x \wedge c y)=T_{2}^{d}(c x \wedge c x)=T_{2}^{d}(c x)=T_{2}(c, x) \\
T_{1}^{d}=T_{2}^{d} \text { for } T_{1}^{d}(c x)=T_{2}^{d}(c x)
\end{gathered}
$$

ii. Since $X$ is semiprime $f$-algebra, $X$ is $d$-algebra. In that case, proof is similar from Theorem 4 . If $T$ is a biorthomorphism on $X$, then $T$ is an orthosymmetric Riesz bimorphism. In other words, if $x \wedge y=0$ on $X$ for $\forall c \in X^{+}, T(c, x) \wedge T(c, y)=0$. If $y=x, T: X \times X \rightarrow X$ defined with $T(c, x)=c x$ is an orthosymmetric Riesz bimorphism. In addition, $X^{d}$ for $y=x=c$ is Riesz space and $X^{d}$ is square of $X$.

Therefore, there is an only Riesz homomorphism $T^{d}: X^{d} \rightarrow X$ defined with $T(c, x)=T^{d}(c x \wedge c y)$ for $\forall x, y \in$ $X$ and $c \in X^{+}$. Moreover $T^{d}$ is an orthomorphism. Indeed, let $|n| \wedge|v|=0$ for $n \in X$ and $v \in X^{d}$. Since $T$ is orthosymmetric, $T(n, v)=0$. On the other hand, there are $x, y \in X$ and $c \in X^{+}$satisfying property $v=c x \wedge$ $c y$ from definition $X^{d}$ for $v \in X^{d}$. From [7] and $\forall c \in X^{+}$for $y=x, n T(c, x)=T(n c, x)=c T(n, x)=$ $T(n, c x)=T(n, c x \wedge c y)=0$ is provided for $T \in \operatorname{Orth}(X, X)$. Since $X$ is semiprime for $y=x$,

$$
|n| \wedge\left|T^{d}(v)\right|=|n| \wedge\left|T^{d}(c x \wedge c y)\right|=|n| \wedge\left|T^{d}(c x)\right|=|n| \wedge|T(c, x)|=0
$$

is obtained. This showed that operator $T^{d}$ is a positive orthomorphism.

iii. For $y=x$,

$$
\begin{aligned}
T^{d}(c x \wedge c y)= & T^{d}(c x \wedge c x)=T^{d}(c x)=T(c, x) \\
& T^{\odot}(c x)=T(c, x)
\end{aligned}
$$

$T^{d}=T^{\odot}$ from $T^{d}(c x)=T^{\odot}(c x)$.

Conclusion 4. Let $X$ is a Dedekind complete $d$-algebra. $\operatorname{Orth}(X)$ is an ordered ideal of $\operatorname{Orth}(X, X)$.

Proof. It suffices to show that $\operatorname{Orth}(X)$ is a solid in $\operatorname{Orth}(X, X) \operatorname{since} \operatorname{Orth}(X)$ is a Riesz subspace of $\operatorname{Orth}(X, X)$, as in the case of $X$ being a Dedekind complete semiprime $f$-algebra [8]. Let $T \in \operatorname{Orth}(X, X)$. By definition of solid, $0 \leq T \leq f$ for $f \in \operatorname{Orth}(X)$. We have to show that $T \in \operatorname{Orth}(X)$. Since $X$ is uniformly complete $d$-algebra, there is an only positive orthomorphism $T^{d}: X^{d} \rightarrow X$ satisfying property $T(c, x)=$ $T^{d}(c x \wedge c y)$ for $\forall x, y \in X$ and $\forall c \in X^{+}$. From here for $y=x=c$,

$$
0 \leq T^{d}(c x \wedge c y)=T^{d}(c c \wedge c c)=T^{d}\left(c^{2}\right)=T(c, c) \leq T\left(c^{2}\right) \leq f\left(c^{2}\right)
$$


is provided. In other words, $0 \leq T^{d}(v) \leq f(v)$ for $\forall 0 \leq v=c^{2} \in X^{d}$. The operator $T^{d}$ has the extension to a positive operator, again denoted by $T^{d}$, which satisfies the property $0 \leq T^{d} \leq f$. From here, $T^{d} \in \operatorname{Orth}(X)$ and $\widehat{T^{d}}=T$ is obtained. Consequently, $T \in \operatorname{Orth}(X)$. This proves that $\operatorname{Orth}(X)$ is a solid in $\operatorname{Orth}(X, X)$ and therefore an ordered ideal.

\section{Author Contributions}

All authors contributed equally to this work. They all read and approved the final version of the manuscript.

\section{Conflicts of Interest}

The authors declare no conflict of interest.

\section{Acknowledgement}

This article was produced from the master thesis [10] prepared by İbrahim GÖKCAN

\section{References}

[1] Aliprantis, C. D., Burkinshaw, O. ( 2006) Pozitive Operators. Springer, Dardrecth.

[2] Benamor, F . (2014) On Bi-orthomorphisms on a Semiprime $f$-Algebra. Indag. Math., 25: 44-48.

[3] Bernau, S. J., Huijsmans, C. D. (1990) Almost $f$-Algebras and $d$-Algebras. Math. Proc. Cambridge Philos. Soc. 107: 287-308.

[4] Birkhoff, G., Pierce, R.S. (1956) Lattice-ordered rings, An Acad. Brasil. Cienc. 28: 41-49.

[5] Birkhoff, G. (1967) Lattice Theory. Amer. Math. Soc. Colloq. Publ., 25, Providence, RI. MR 372638.

[6] Boulabiar, K., Brahmi, W. (2016) Multiplicative Structure of Biorthomorphisms and Embedding of Orthomorphisms. Indag. Mathem, 27(3): 786-798.

[7] Buskes, G., Rooij, A. (2000) Almost $f$-Algebras: Structure and the Dedekind Completion. Positivity, 4: 233-243.

[8] Buskes, G., Page, R. and Yilmaz, R. (2010) A note on bi-orthomorphisms. Oper. Theory Adv. Appl., 201: 99-10.

[9] De Pagter, B. (1981) $f$ - algebras and orthomorphisms. Phd. Thesis, University of Leidin.

[10] Gökcan, İ. (2017) Multiplicative Structure of Biorthomorphisms and Relation with Orthomorphisms. Master Thesis, Recep Tayyip Erdoğan University.

[11] Huijsmans, C.B. (1991) Lattice ordered algebras and $f$-algebras. A survey. Studies in Ekonomic Theory 2, Positive Operators, Riesz Spaces and Economics (C. D. Aliptantis, K. C. Border and W. A. J. LuAemburg, eds.) Spinger, Berlin, 151- 169. 
[12] Kudlacek, V. (1962) On some types of l -rings. Sborniysokehovceni Techn v Brne, 1-2: 179-181.

[13] Kusraev, A. G., Tabuev, S. N. (2008) Multiplicative Representation of Bilinear Operators. Siberian Mathematical Journal, 49(2): 287-294.

[14] Zaanen, A. C. (1975) Examples of othomorphism. J. Approximation theory, 13: 192-204. 